\title{
Studying Differential Invariants in Developmental Variations
}

\author{
Jacques Juhel \\ Univ Rennes, LP3C (Psychology Laboratory: Cognition, Behavior, Communication), Rennes, France \\ Email: jacques.juhel@univ-rennes2
}

How to cite this paper: Juhel, J. (2018). Studying Differential Invariants in Developmental Variations. Psychology, 9, 655-678.

https://doi.org/10.4236/psych.2018.94041

Received: March 2, 2018

Accepted: April 15, 2018

Published: April 18, 2018

Copyright $\odot 2018$ by author and Scientific Research Publishing Inc. This work is licensed under the Creative Commons Attribution International License (CC BY 4.0).

http://creativecommons.org/licenses/by/4.0/

\begin{abstract}
The differential study of intraindividual variability and change is currently reaping the benefits of important methodological advances in longitudinal data modeling. Two major research strategies for detecting invariants in individual differences in developmental change are considered. The most common approach involves operating at a population level to study differences of behavior throughout development. Though rarely used, another approach is the differential study of developmental change based on an individual modeling of the dynamics of intraindividual variability. The potential of these two approaches for improving our understanding of the development of human beings is illustrated.
\end{abstract}

\section{Keywords}

Individual Differences, Intraindividual Variability, Longitudinal Data Modeling

\section{Introduction}

Broadly defined, psychology aims to understand the psychological mechanisms underlying behavioral invariance in its various manifestations. The study of invariance first requires an "abbreviated" description of the diversity of psychological phenomena (Reuchlin, 1962). This description can be a simple summary of phenomena to which a property is then assigned. An invariant can also be defined using abstract principles designed to account for specific phenomena (e.g. a learning rule, a structure of correlations supporting a hypothesis, or a schema specifying the operative invariance of a form of reasoning at a given age). The aim is to specify the (invariant) principles linking variations in behavior in different situations, either in different individuals or at different moments in time, with a view to determining whether a hypothesis is confirmed by data or making 
predictions based on these data.

\subsection{Regularities, Stabilities and Variabilities in Temporal Variations}

In order to provide evidence of invariance in change, the regularities and stabilities characterizing temporal variations need to be identified. The search for invariants will be determined by the particular types of variability selected for analysis. In developmental psychology, the long dominant strategy involved comparing groups of individuals of different ages in similar circumstances (cross-sectional approach) or comparing different periods of development in the same individuals (longitudinal approach) based on samples of interchangeable individuals. The aim was to highlight broad trends and average "patterns" of change in the population, such as common learning and development in children at a given age, epistemic regularities in development or general laws governing development.

Comparisons of groups of individuals of the same age or comparisons of different periods of development in the same individuals can be further supported by research on interindividual differences. Here, the aim is to identify laws of variability. Differentiating individuals implies classifying them on the basis of their distance from the "center" of the group, which explains why studies in this area commonly refer to average group trends. However, the use of interindividual comparisons requires that interindividual variations show a degree of temporal stability, an idea implied by the idea of the "dependability" or temporal stability of scores in a test performed at close intervals (Cattell, 1964). Other forms of temporal stability or continuity have also been studied in differential psychology. For example, differential stability is the degree of stability in the relative ranking (or rank-ordering) of individuals over longer periods of time (at least two months, according to Cattell, Eber, \& Tatsuoka, 1970). Differential stability implies that there are no interindividual variations in intraindividual change (Asendorpf, 1992). Structural continuity (another form of temporal stability or continuity) can be defined as the structural stability of interindividual differences at different moments in time. A persistent and hierarchical relationship between complementary dimensions at an intraindividual level (for example, between positive and negative emotions) implies ipsative stability (Brim \& Kagan, 1980). In addition to the various types of homotypic continuity, research has also focused on heterotypic continuity (Kagan, 1971). Heterotypic continuity is inferred from correlations between different behavioral manifestations throughout development (e.g. the relationship between a young child's difficult temper and the older child's aggressiveness) that are theoretically similar (in this case, reactive and negative emotionality; Bates \& Novosad, 2005).

It is thus clear that the various types of temporal continuity or stability defined in differential psychology primarily involve the question of temporal invariance in the measurement of interindividual differences and the latent structure 
underlying these differences. However, the differential analysis of developmental variables or the temporal evolution of the structure of the variables emerging from differences between individuals requires an examination of the sources of variation located at different levels of analysis.

\subsection{Levels of Analysis in Temporal Variations}

In dealing with psychological and behavioral regularities and similarities, extracting invariants requires identifying properties indicating uniformity or homogeneity and applying to all units at a particular level of analysis. This chapter focuses on invariants in differences in developmental change. The chosen level of analysis is located between the universal and individual levels. Each level of analysis is associated with a specific type of comparison.

An invariant is universal if it represents the characteristics of every individual in a population. The search for an invariant in developmental change requires comparing entities based, for example, on comparisons of two groups of children of different ages or of different moments in the same individuals observed over time. For example, motor learning and language development are universal invariants that have a representative value which can be used to determine what children of a given age have in common (e.g. first steps at around 11 months, first use of relative pronouns and conjunctions at around 5 years of age).

An invariant is differential if it resides in the properties or characteristics of a stable differentiation between subpopulations (for example, on average, girls say their first word at a younger age than boys) or between individuals within a population (for example, differences in working memory performance are correlated to differences in reading comprehension among young readers). The search for invariants in differences in developmental change requires an analysis of the relationships between measures of the same variable at different moments in time. The analysis of persons $\times$ occasions data (Cattell, 1952) can be used to study different invariants such as the latent structure of interindividual differences in developmental change or the rate of developmental change per unit of time.

The search for invariants at a universal or differential level involves making a set of observations at different times among different individuals. The behavior of each individual is then compared to a trend (i.e. means, variances, covariances, etc.). In this sense, the various methods of analysis applied to data obtained by aggregation of individuals may be said to be normative. The information provided by the statistical results of these analyses applies to the population as a whole, from which individuals are randomly selected. The interpretation of this information is relevant for the study of developmental variables, the relationships between these variables and the factors that have an effect on these variables, on change and on interindividual differences in developmental change.

Unless it can be shown that what holds true for the population also holds true for the individual (on this point, see Molenaar, 2004; Molenaar \& Campbell, 
2009), the analytical framework outlined above is not premised on the type of hypothesis typically found in a theory explaining developmental processes at an individual level. Here, the appropriate level of analysis is the individual, based on comparisons of observations at different moments in time. A study of intraindividual variations is required to identify invariants accounting for the idiosyncrasies of individual development. The scheme defined by Vergnaud (2007) as an invariant form of organization of activity based on the experiences of the individual and varying according to the specific characteristics of the situation is an example of this type of individual invariant. Another individual type of invariance is the structure of covariation between variables measured at different moments in time in the same individual.

The three levels of analysis of developmental change provide different information. To focus exclusively on one level is to run the risk of assigning the responsibility of the observed variations to the causes of variations located solely at this level of analysis. For example, it may be true that differences in developmental change observed in children over a given period of time reflect the average change observed at a population level. However, these differences may be partly due to interindividual variations in systematic intraindividual variations, with the development of some children differing more from the average level of developmental change than the development of other children. Variations may also be due in part to random intraindividual variations in developmental change. Therefore, it is important to ensure that the different levels of analysis complement one another. One example of the potential complementarity of universal analysis and differential analysis is the incorporation into the Piagetian model of the analysis of inter- and intraindividual differences associated with the hypothesis of a plurality of adaptive processes, organized into systems and varying between individuals and at different moments in time (Reuchlin, 1978). The pluralistic and multidimensional model of cognitive development proposed by Lautrey $(1990,1993,2003 a)$ is another good example of this approach. The evolution of the epistemological status of variations (Lautrey, Mazoyer, \& van Geert, 2002; Lautrey, 2003b, 2010; Reuchlin, 1999) and the progress made in the analysis of intraindividual change and the individual analysis of the temporal dynamics of intraindividual change are also indicative of the potential for combining individual and differential levels of analysis of developmental change.

\section{The Search for Differential Invariants in Developmental Change Based on a Normative Analysis of Intraindividual Change}

Intraindividual variations are a key focus of current research on developmental change. The study of intraindividual variations is based on a classical taxonomy involving three different types of intraindividual change based on continuous variations at different moments in time (Nesselroade, 1991). The concept of intraindividual change applies to irreversible changes in learning and develop- 
ment. The assumption is that gradual changes are cumulative (e.g. numeracy learning and language development) and can be observed over time (long-term perspective). The concept of intraindividual variability applies to more or less reversible changes that are synchronous or asynchronous among individuals, based on a real-time perspective (e.g. spontaneous variations, cyclical fluctuations and variations in response to a change in constraint; Fiske \& Rice, 1955). Nesselroade (1991) illustrated the relationships between intraindividual variations in real time and in the longer term by comparing the developmental process to the production of a fabric, including the warp (vertical threads corresponding to intraindividual change) and the woof (horizontal threads corresponding to intraindividual variability). A third type of intraindividual variability is the distribution of measurements performed on the same individual undergoing several tests on the same occasion (for example, the standard deviation of the distribution of scores). The individual pattern of functional organization (or scatter) is characterized by salient intraindividual characteristics. However, changes over time in the intraindividual pattern can only be envisaged at an individual level of analysis. Such a diverse representation of intraindividual variability requires an account of the theoretical relevance of the different types of variability according to the studied phenomenon of change.

Studies of intraindividual variability and change are generally conducted at a population level, and more rarely at an individual level. The approach is known as "variable-oriented" (i.e. comparison of different age groups or of individuals at different times) when each datum draws its meaning from its position in relation to the data of the group on which the variable is measured. Here, the focus is on changes over time in the average position of the group and/or the relative position of individuals. In the "person-oriented" approach, the aim is to empirically characterize an individual based on a profile of intraindividual variability established on the basis of a configuration of variables measured at different moments in time (Bergman, Magnusson, \& El-Kouri, 2003). Here, the study of developmental change involves examining the temporal evolution of profiles of intraindividual variability shared by individuals. In both of these normative approaches, the data may come from cross-sectional or longitudinal designs or from longitudinal measurement-burst designs in which each person is assessed multiple times on each occasion (Nesselroade, 1991). However, researchers in developmental psychology have long recognized that although they are difficult to apply, longitudinal designs are the best method for empirically assessing theoretical hypotheses about developmental change and interindividual differences in change.

Research on developmental change over the lifespan (Baltes, Reese, \& Nesselroade, 1977), as pioneered by Nesselroade (1970), is an example of the normative approach (in the sense defined above) of intraindividual change. In these types of studies, observation data are generally collected from a large sample of individuals observed at different times (two or three times, sometimes 5 or 6 , 
rarely more), then aggregated across individuals at each moment. The aim is to characterize intraindividual change, to identify differential invariants in intraindividual change and to analyze the relationships between indicators of change and the antecedents, consequences and correlates of intraindividual change and interindividual differences in intraindividual change (Baltes \& Nesselroade, 1979). In recent years, there has been a gradual shift to a search for more dynamic invariants such as changes in trajectory or developmental transitions (Nesselroade \& Ghisletta, 2000).

The results obtained using statistical methods to unravel different types of variability (see below for a discussion of these methods) are indicative of the value of the chosen approach. When they are sufficiently significant from the point of view of intraindividual change, the different types of real-time variations represent a significant proportion of variance compared to interindividual variations (up to half, according to Nesselroade \& Salthouse, 2004). Some indicators of the scale of aggregated intraindividual variability, i.e. calculated without taking into account the temporal structure of variations (for example, intraindividual standard deviation or the individual mean of the squares of the differences between successive observations), can be used to predict other interindividual characteristics, which demonstrates their validity. When isolated from other types of variability, intraindividual variability may even suggest a new explanation. For example, an asynchronous increase in intraindividual variability in cognitive performance in elderly subjects may contribute to explaining an increase in interindividual variability in cognitive performance compared to younger subjects (Nesselroade \& Ram, 2004).

However, theoretical interpretations of intraindividual variability can be ambiguous. For example, in some circumstances, intraindividual variability can be given a theoretically coherent interpretation. In this case, we speak of functional or adaptive intraindividual variability or of maladaptive intraindividual variability. However, intraindividual variability may also be unrelated to behavior or affected by measurement errors (Schmidt, Le, \& Ilies, 2003). In some cases, intraindividual variability serves as a predictor of, or a necessary condition for, developmental change, while at other times it is a manifestation or result of change (Martin \& Hofer, 2004). For example, intraindividual variability measured before a developmental transition is weakly related to intraindividual variability measured after the transition. The increase in behavioral flexibility throughout child development is accompanied by an increase in behavioral consistency, i.e. a decrease in intraindividual variability.

Although the methods used in longitudinal data analysis are particularly well-suited to identifying invariants in developmental change, it is important to ensure that the developmental hypotheses are consistent with the statistical models used to test them. Consider, for example, the study of the development of working memory in children initially assessed at 3 years of age and subsequently once a year up to the age of 7 . The hypothesis of a more rapid develop- 
ment of working memory at the end of the observation period can be examined using a regression model describing changes (for example, quadratic changes) in the performance of children from the first to the fifth measurement. However, from a theoretical point of view, this hypothesis requires homotypic continuity or a constant rate of change over time across the sample. Therefore, provided the collected data allow for it, the use of a statistical model to explicitly test these different aspects significantly increases the overall consistency. The translation of a developmental hypothesis into a formal data generation model is also a key stage that is dependent upon how the development process is conceived. If development is conceived as a continuous process, e.g. as being synonymous with linear growth, the models used will approximate the longitudinal measurements performed by a non observable continuous trajectory. The modeled data are often quantitative (continuous or count data), but may also be qualitative (ordinal or categorical data) or relate to the occurrence of an event (censored data). Developmental change can also be viewed as a discontinuous process synonymous with a qualitative change. In this case, modeling the developmental process will involve a direct or indirect measurement of successive states and a description of the dynamics of transition between these states. These models are generally applied to categorical data, but can also be applied to quantitative data. In short, in either one of these approaches, there are many statistical models for identifying differential invariants in developmental change.

\subsection{The Differential Study of Individual Developmental Trajectories}

The methods used to analyze developmental trajectories differ according to the chosen level of analysis (universal or differential) and the nature (quantitative or qualitative) of the interindividual differences examined (Bauer \& Reyes, 2010; Curran \& Bauer, 2011).

The simplest hypothesis assumes that all individuals in the population have the same developmental trajectory. In a time-dependent linear regression model:

$$
y_{t}=\beta_{0}+\beta_{1} x_{t}+\varepsilon
$$

$\beta_{0}$ and $\beta_{1}$ represent, respectively, the mean intercept and the average linear slope (the rate of change per unit of time) in the population, with the index $t$ indicating time. Both fixed parameters have the same value for all individuals in the population (see Figure 1(a)).

The traditional method used to reach the differential threshold is to hypothesize that all individuals have the same form of latent developmental trajectory by freeing the growth trajectory parameters, i.e. by allowing them to vary in different individuals (see Figure 1(b)). Multilevel methods for the simultaneous modeling of interindividual differences and intraindividual change (Bryk \& Raudenbush, 1987; Collins, 2006; Gibbons, Hedeker, \& DuToit, 2010; Raudenbush \& Bryk, 2002) or latent growth modeling (LGM) (Bollen \& Curran, 2006; McArdle \& Epstein, 1987) can be used to address the issue of interindividual differences in latent developmental trajectories. 


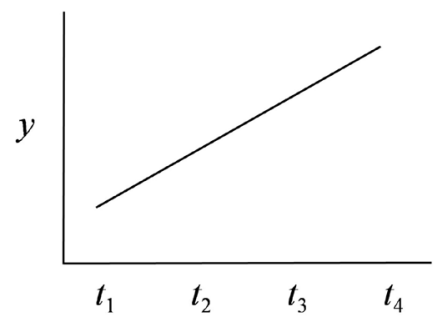

(a)

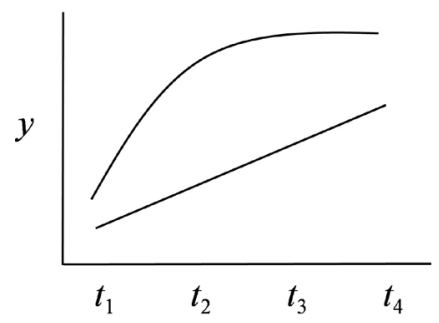

(c)

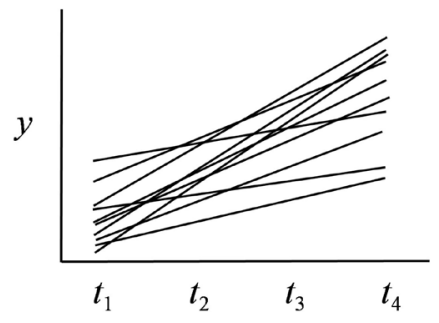

(b)

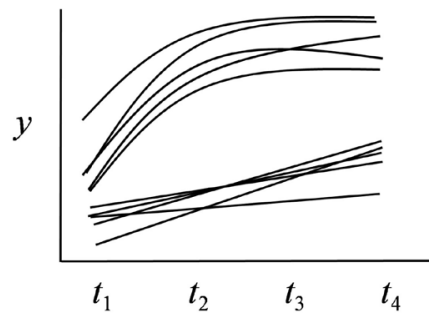

(d)

Figure 1. Population-level analysis: (a) Linear Regression (LR) on an outcome over time; (b) Mixed Model, MultiLevel Growth Model (MLGM), Latent Growth Modeling (LGM) or Latent Growth Curve analysis (LGC): Quantitative interindividual differences between individual trajectories on an outcome over time; (c) Group-Based Trajectory Modeling (GBTM) or Latent Class Growth Analysis (LCGA): Qualitative differences between clusters of individuals following similar trajectories on an outcome over time; (d) Growth mixture Modeling (GMM): Qualitative differences between clusters of individuals following similar trajectories over time and quantitative interindividual differences within clusters.

The most general model is based on the hypothesis of a potentially correlated random intercept and slope, although it can have a random intercept only or a random slope only. It is expressed as follows:

$$
\begin{array}{r}
y_{t i}=\beta_{0 i}+\beta_{1 i} x_{t i}+r_{t i}, \\
\beta_{0 i}=\gamma_{00}+u_{0 i} \\
\beta_{1 i}=\gamma_{10}+u_{1 i}
\end{array}
$$

where $\gamma_{00}$ and $\gamma_{10}$ represent the mean intercept and the mean slope and $u_{0 i}$ and $u_{1 i}$ are the individual deviations from the mean intercept and the mean slope, with the index $i$ referring to the individual.

By grouping Equation (2), describing systematic intraindividual change replacing the development process, and Equations ((3) and (4)), which describe interindividual variations around growth parameters, a reduced version of the mixed model is obtained:

$$
y_{t i}=\left(\gamma_{00}+\gamma_{10} x_{t i}\right)+\left(u_{0 i}+u_{1 i} x_{t i}+r_{t i}\right)
$$

where the distinction between fixed effects (the same across the population) and random effects (different in different individuals from the same population) is clearly apparent. The hypothesis of normally distributed individual intercepts and slopes provides a summary of the quantitative differences between individu- 
als with fixed means (the mean intercept, the mean slope) and freed variances (interindividual variance around the mean intercept and around the mean slope). Covariables can also be introduced as predictors or correlates of interindividual variations. For example, based on a sample of children aged 6 to 11 years observed annually on four consecutive occasions, Kim and Cicchetti (2006) showed that the level of emotional and physical maltreatment could be used to positively predict the initial depression score and to negatively predict the annual rate of linear decline in depressive symptoms.

Despite their usefulness in describing change in relation to time, the models presented above do not provide a direct representation of intraindividual changes. They posit that individual parameters remain stable over time (variance of the intercept and of the slope or slopes, the covariance(s) between intercept and slope(s)), meaning that they are stationary models at an intraindividual level. A more dynamic account of intraindividual change involves using autoregressive models with latent variables in which changes in a variable $t$ are a function of the same variable at time $t-1$ and of one or several other variables at an intraindividual level. This approach, known as coupled change (Sliwinski \& Mogle, 2008), combines multilevel modeling of change and the modeling of the dynamics between several processes. It can be used to test hypotheses about lagged relationships among latent variables measured on $\mathrm{t}$ occasions. McArdle $(2001,2009)$ provides an overview of dynamic structural models known as latent change score models. Ferrer et al. (2007) used these models to test the hypothesis of a positive relationship between the fluid component of intellectual performance and the development of academic knowledge from childhood to adolescence. Ferrer et al. (2010) showed that the range of dynamic relationships between reading and cognition was higher between the ages of 7 and 9 than at older ages and that, unlike good readers, cognition and reading in dyslexic children are not mutually coupled throughout development.

The interindividual variations examined in the methodological framework described above were quantitative. However, the hypothesis of qualitative interindividual differences also needs to be examined when a single trajectory fails to capture all individual trajectories. Based on typical latent trajectories, a latent class measurement model can be used to represent the qualitative differences between a small number of subpopulations of individuals with the same growth curve (for example, linear for one class, logarithmic for another, etc.). Latent class analysis (Lazarsfeld \& Henry, 1968) provides a comprehensive account of interindividual variability based on a limited number of non-observable homogeneous subgroups or classes in the population. Latent classes are inferred from response patterns over time, just as a continuous growth factor is inferred from covariations between responses over time. The probability of an individual belonging to one of these classes is also considered.

The taxonomic part of the model can be interpreted with varying degrees of psychological realism. The purely qualitative approach focuses merely on the 
form of developmental trajectories and does not require modeling interindividual variability in each latent class. The model posits that each class of individuals is homogeneous in terms of the process of change (group-based trajectory modeling, or GBTM; Nagin, 2005; Nagin \& Odgers, 2010) (see Figure 1(c)). Another assumption is that trajectories vary between individuals from the same subpopulation (see Figure 1(d)). In this case, the model hypothesizes that there are continuous individual intercepts and slopes distributed normally in each class (growth mixture modeling, or GMM; Muthén, 2004). Compared to the purely qualitative approach, this method confers a greater degree of psychological reality to the latent classes, the members of which are no longer interchangeable, though it is also controversial since it is always possible, in the absence of population heterogeneity and under certain conditions, to distinguish subgroups of individuals (see, in particular, Bauer \& Curran, 2003). Regardless of the chosen approach, the interpretation of these classes requires connecting interindividual variations in growth trajectories to temporally invariant predictors, covariables varying in time or distal criteria.

The value of this family of mixed distribution models can be illustrated by the results of a study aimed at identifying children with reading difficulties. Based on a sample of children followed from preschool to the third year of primary school, Boscardin et al. (2008) administered tests measuring two predictors of reading performance (phonological awareness and naming speed) and a reading test (word recognition). The tests were administered four times a year over a three-year period (12 times in total). The study examined the development of phonological awareness in preschool children (4 measurements) and the development of word recognition in the same children in the two following years (8 measurements). Based on a growth-mixture model (see Figure 1(d)), five developmental profiles were identified (phonological awareness). Five other developmental profiles were also identified in the two following years (word recognition). The final model combined the model of phonological awareness development and the model of word recognition development. Naming speed measured at the end of kindergarten was used as a predictor of the classification of the developmental trajectories of phonological awareness at preschool age on the one hand and of the developmental trajectories of word recognition between the ages of 7 and 8 on the other. Among other things, the results showed that faster naming speed is associated with better reading skills and that preschool children in whom phonological awareness develops more slowly make slower progress in reading between the ages of 7 and 8 . Evidence of differences in the developmental profiles of students with similar reading difficulties highlights the need for a multivariate approach to reading difficulties (the "complex-interactions principle"; see Sterba \& Bauer, 2010).

In short, the models used to study individual trajectories have changed significantly in recent years. The analysis of individual trajectories initially focused on variables to study time-correlated continuous change and/or on the structures of 
variables to examine more dynamic aspects of change. Subsequently, the focus shifted to identifying typical intraindividual profiles (Sterba \& Bauer, 2010; von Eye, 2010; von Eye \& Bergman, 2003) by incorporating growth curve analysis and latent class analysis in the same model (Muthén, 2008). These models have the advantage of not automatically locating all individuals on the same quantitative level. Accordingly, they involve a shift of focus to a more qualitative description of interindividual differences in the mechanisms underlying developmental change (see the notion of vicariance or substitution between more or less adaptive behaviors, processes or strategies; Reuchlin, 1978). A similar shift can be seen in the application of the methods of differential psychology to the study of discontinuous developmental change.

\subsection{The Differential Study of Qualitative Change over Time}

When developmental change involves qualitatively different phases or stages, it is necessary to use models for the analysis of change that formalize the membership of individuals to a finite number of subpopulations at every moment in time. Here, developmental change is represented by transitions through discrete categories. The assumption is that a transition from one category to another is indicative of a qualitative change. The general approach is based on Markov chain models and their latent variable extensions (Langeheine, 1988; Langeheine \& van de Pol, 1990). The following is partly based on Kaplan $(2008,2009)$.

The simplest model of qualitative change for categorical longitudinal data is the manifest Markov model. This autoregressive model involves a chain in which the state of development measured at time $t$ by an indicator is completely predicted by the measurement performed at time $t-1$ (see Figure 2(a)). It is expressed for a categorical variable measured (for example) at four different moments at equal intervals:

$$
P_{a b c d}=\delta_{a}^{1} \tau_{b \mid a}^{21} \tau_{c \mid b}^{32} \tau_{d \mid c}^{43},
$$

where $a, b, c$ and $d$ are the values of the categories of the variable measured at times $1,2,3$ and 4 , with $a=1, \cdots, \mathrm{A}, b=1, \cdots, \mathrm{B}, c=1, \cdots, \mathrm{C}$, and $d=1, \cdots, \mathrm{D}$. $P_{a b c d}$ is the predicted proportion of individuals showing the pattern of responses $(a, b, c, d)$. The parameter $\delta_{a}^{1}$ is the observed proportion of individuals in each of the a categories of the variable measured at time 1 . The parameters $\tau_{b \mid a}^{21}, \tau_{c \mid b}^{32}$ and $\tau_{d \mid c}^{43}$ are the transition probabilities corresponding to the dynamic part of the model. For example, $\tau_{b \mid a}^{21}$ is the transition probability from time 1 to time 2 for individuals in category $b$, based on the assumption that they were in category $a$ at time 1 . In the case of an observed dichotomous variable, the matrix of transition probabilities from time 1 to time 2 contains four probabilities: the probability of observation 0 at time 2 having observed 0 at time 1 ; the probability of observation 0 at time 2 having observed 1 at time 1 ; the probability of observation 1 at time 2 having observed 0 at time 1; and the probability of observation 1 at time 2 having observed 1 at time 1 (Figure 2(a)). Transition probabilities may remain constant over time (stationary Markov chain), implying a decreased 
(a)

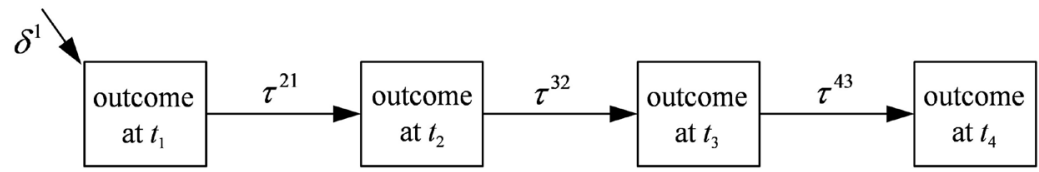

(b)

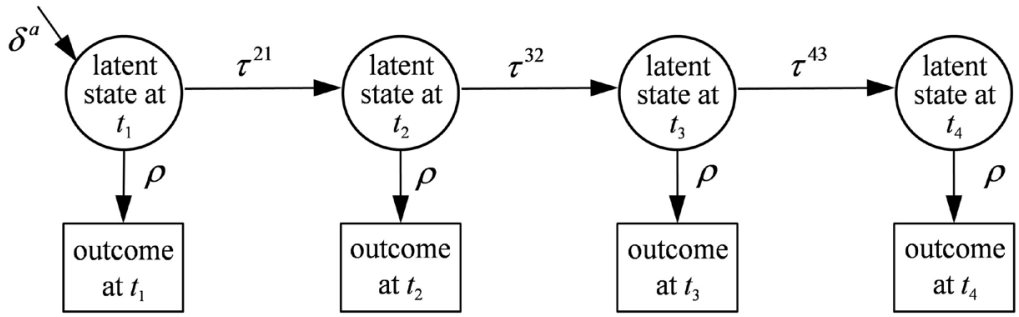

(c)

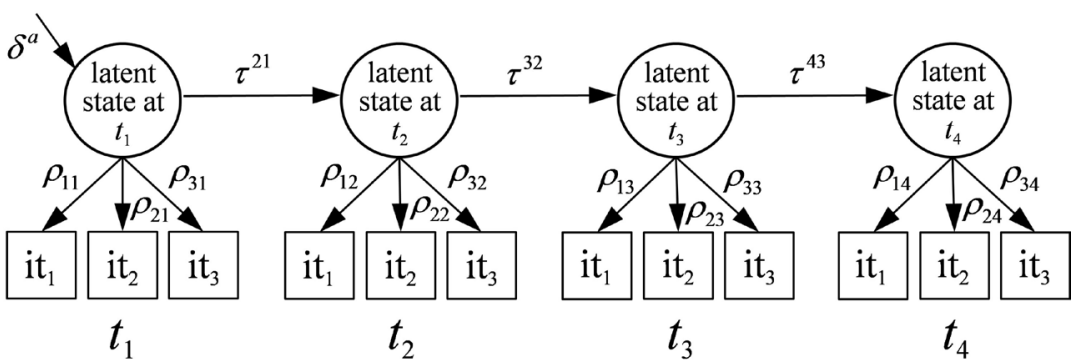

(d)

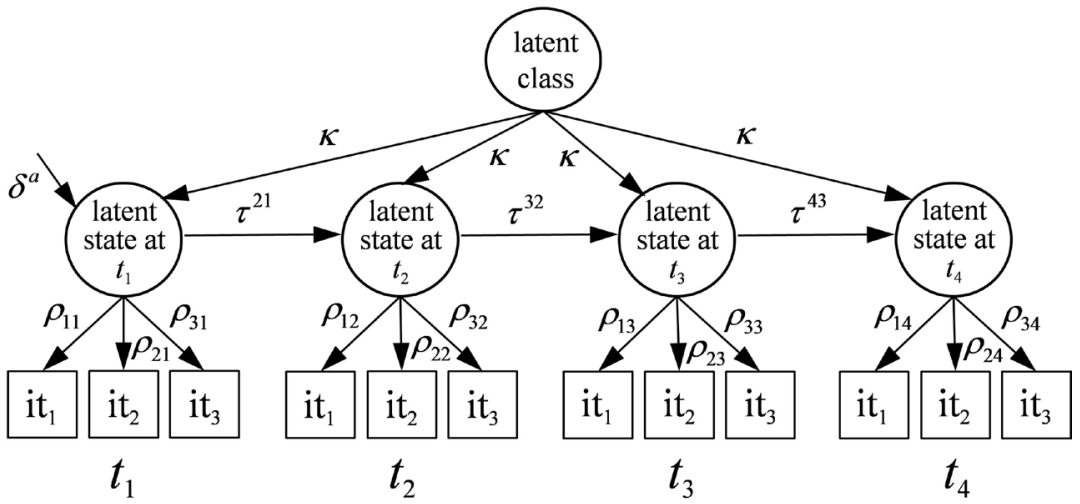

Figure 2. Markov models and latent class analysis for discontinuous change: a) Manifest Markov process: transition probability $\tau$ between observed categories; b) Hidden Markov model (HMM): transition probability $\tau$ between latent states; probability $\rho$ of being in the observed category, knowing the latent state; c) Latent Transition Analysis (LTA): transition probability $\tau$ between latent states; probability $\rho$ of being in the observed category, knowing the latent state; d) Mixture Latent Transition Analysis (MLTA): proportion $\kappa$ of individuals in each subpopulation; transition probability $\tau$ between latent states; probability $\rho$ of being in the observed category, knowing the latent state.

association between measurements at different moments in time. They may also be freed, meaning that the dynamics of the model change over time (non-stationary Markov model).

The hidden Markov model (HMM) adds a latent variable to the Markov 
process (a discrete distribution with several latent states), which measures the indicator without error. The estimated parameters are the probabilities of observing the response of an individual in a given category, conditional on the latent state and the transition probabilities between latent states (see Figure 2(b)). Their estimation requires that the response probabilities over time be constrained to invariance. By combining the Markov chain model and the latent class model in which the latent state is measured by several indicators at several points in time, we obtain Markov multiple indicators models (van de Pol \& Langeheine, 1990) or latent transition analysis (LTA; Collins \& Lanza, 2010) (see Figure 2(c)). Here, the aim is to group individuals with the same profile of change, i.e. individuals whose latent state changes in the same way over time. This method can be used to estimate common trajectories through successive latent states and to explore trajectory differences between different subgroups from the same population. Quaiser-Pohl et al. (2010) examined the evolution of solution strategies in a mental rotation task (16 dichotomous items) based on a sample of 296 children aged 5.5 years (on average). The children were studied on two separate occasions, with 104 children undergoing a training period between the two measurements. Three stages of development were identified: before 60 months (use of inappropriate strategy), around 65 months (use of semiappropriate strategies) and at 70 months (use of analytic or holistic appropriate strategies). The range of solution strategies used by the children is summarized in six latent classes. Multigroup latent transition analysis showed that there were differences in the dynamics of transition between groups. For example, there were a greater proportion of children moving toward an appropriate strategy in the trained group compared to the control group.

In latent transition analysis, the sample is drawn from a population with one Markov chain, although there may be several unobserved subgroups within the population. The hypothesis of latent heterogeneity is similar to the hypothesis outlined above, where the same continuous trajectory failed to account for qualitative differences between different trajectories of change. The solution is the same. The assumption is that each subgroup is characterized by a latent Markov chain with different parameters from one subpopulation to another. The corresponding model is known as mixture latent transition analysis (MLTA) (see Figure 2(d)). A particular case of this model is the mover-stayer model, which posits that the latent status changes over time in some individuals (for example, individuals who go from one strategy to another) and not in others (for example, individuals who always use the same strategy). However, this family of models is rarely used in the differential study of developmental change (for an example in the area of reading acquisition, see Kaplan, 2008; Kaplan \& Walpole, 2005).

The models outlined above are particularly useful for studying discontinuous developmental processes, for grouping individuals whose latent status changes in the same way over time and for identifying qualitatively different profiles of intraindividual change (Bray, Lanza, \& Collins, 2010; Collins \& Lanza, 2010; 
Muthén, 2008). They are particularly useful in person-oriented research, especially in view of the flexibility of currently available statistical tools (for example, Mplus: Muthén \& Muthén, 1998-2017).

\section{From the Individual Study of Structures of Intraindividual Variation to the Identification of Differential Invariants in Developmental Change}

The methodological framework outlined above can be used to examine quantitative and qualitative differences in developmental change at a population level. The assumption is that developmental processes are temporally homogeneous and that the behavior of individuals from the same population is governed by the same dynamic laws. However, recent studies in this area have not generally complied with the hypothesis of the ergodicity of developmental processes (Molenaar, 2004; Molenaar \& Campbell, 2009). A developing system responds differently in different contexts and changes over time as a result of its own activity. Therefore, the underlying dynamic laws do not present the statistical characteristics of temporal invariance (i.e. constant mean, variance and sequential dependence over time) implied by the normative approach to developmental change. An approach that is more compatible with the non-ergodicity of developmental processes involves modeling intraindividual variability at an individual level, a fundamental measure of change and the "intellectual parent of dynamic systems analysis in psychology" (Boker, Molenaar, \& Nesselroade, 2009: p. 858).

The distinction between the normative analysis of intraindividual change and the modeling of intraindividual variability at an individual level is similar in some respects to the distinction between the nomothetic approach (the dominant approach in scientific psychology) and the idiographic approach (Allport, 1937). However, individual-level analysis, which, according to Cattell (1966), is appropriate for the study of dynamic structures, is not based on the assumption that every individual is unique and incomparable. Rather, it involves an individual approach "à la Wundt" (Lamiell, 1981; Molenaar, 2004) that capitalizes on individual differences in the structure of intraindividual variability. Thus conceived, an individual-level analysis of developmental processes avoids the ecological fallacy ("what is true of the population is also true of the individual"; Robinson, 1950) and of the individualistic error ("what is true of the individual is also true of the population"). The aim of the individual-level approach to intraindividual variability is to identify more general psychological and behavioral regularities based on a coherent and rigorous analysis of the similarities and differences between structures of intraindividual variation (Nesselroade \& Molenaar, 1999; Nesselroade, 2010). The aim is to achieve a rapprochement between the normative and individual levels based on an aggregation governed by "the differences and similarities in intraindividual patterns of variability" (Nesselroade, 2004: p. 228) of the individual data. The informed grouping of in- 
dividuals in whom similar structures of intraindividual variation have been found thus appears to be a second avenue for detecting interindividual invariance in intraindividual change.

This idea had already been discussed by Stern in a book published in 1911. However, the idea of studying developmental change at an individual level on a scientific basis has had little influence on research practices in developmental psychology. The main reason for this has to do with the difficulty of measuring the behavior of the same child several times over a relatively long period (see, nevertheless, Van Dijk \& Van Geert, 2007). We now have a partial solution to this issue that involves using computerized methods for collecting intensive observations (Moskowitz et al., 2009). Intensive measurements improve the accuracy and reliability of indicators and can be performed in a laboratory setting or in a natural environment (for example, the experience sampling method and ecological momentary assessment; Ebner-Priemer \& Trull, 2009). However, although microgenetic studies can involve intensive measurements performed at the time scale of development, recent studies have generally been based on data collected in real time. The prospects offered by this type of approach nevertheless warrant a brief examination of various methods for describing the temporal dynamics of intraindividual variability at an individual level.

P-technique factor analysis was originally proposed by Cattell (Cattell, Cattell, \& Rhymer, 1947) and provides a representation (at an individual level) of intraindividual variations of several variables measured over time and of their covariations. Each variable is associated with a time series-in other words, a series of sequential data obtained for the same individual at different moments in time. The aim is to identify the common factor structure that best explains the intraindividual covariance structure. Operating at an individual level, this invariant can be seen as an indicator of individual dynamic characteristics.

Quinn \& Martin (1999) used P-technique factor analysis to examine the structural invariance of intraindividual variations of negative mood in 8 women aged 60 and 80 years. Three dimensions of negative mood (anxiety, stress and fatigue) were measured using 3 items to which the participants responded every day over a period of one hundred consecutive days. P-technique factor analyses are only applied to individual data presenting a sufficient degree of intraindividual variability to enable analysis-in this case, 4 of the 8 participants. The results show that two dimensions are sufficiently significant to account for intraindividual variations of negative mood in the participants aged 60 and 80 years. However, the interpretation of these dimensions varies according to age: anxiety and fatigue vs stress in the two participants aged 60 years and fatigue vs stress in the participants aged 80 years. The authors noted that there was no evidence of the anxiety dimension in the two oldest participants, with very few intraindividual variations in this area compared to those observed in the two youngest women. The authors also found that intraindividual variations in the fatigue dimension were more related to the individual factor than to the effect of age. 
These findings suggest that the value of isolated observations in the study of individual differences in developmental change needs to be reassessed.

Various dynamic extensions of this approach have since incorporated multivariate time series analysis in P-technique factor analysis to take account of the dependence between successive observations (Molenaar, 1985; Molenaar, de Gooijer, \& Schmitz, 1993; Nesselroade, McArdle, Aggen, \& Meyers, 2002; Nesselroade \& Molenaar, 1999, 2004). Models focusing on the interdependence and dimensionality of intraindividual processes are known as dynamic factor analysis (DFA) models (Ram, Brose, \& Molenaar, 2013). A dynamic factor analysis model incorporates the distinction between the observed variables and the continuous factors (internal state). It includes a measurement equation (or observation equation) and one or several transition equations describing the dynamics of the system. In the simplest case, the measurement equation is expressed as follows:

$$
y_{t}=\Lambda \eta_{t}+\varepsilon_{t},
$$

where the vector of the observed variables $y_{t}$ is generated by the latent variables $\eta_{t}$ and the residuals $\varepsilon_{t} \Lambda$ is the factor loading matrix.

The dynamics of the system can be modeled in different ways. One model posits that previous latent characteristics influence subsequent manifestations. This model hypothesizes that the factors have direct effects (staggered in time) on the observed variables (Molenaar, 1985). In this case, the factors are randomly distributed in the non-stationary time series (impact model; white noise factor score model, or WNFS; Nesselroade et al., 2002). Another model, proposed by McArdle (1982), is based on the idea that the latent characteristics at time $t$ subsequently influence these same characteristics. Here, the model is a direct autoregressive factor score model, or DAFS (Nesselroade et al., 2002). The time series of factors is viewed as a stationary process inducing a time series of manifest variables. In the case where the latent variables at time $t$ are generated by themselves at previous points in time, the transition equation is expressed as follows:

$$
\eta_{t}=B_{1} \eta_{t-1}+B_{2} \eta_{t-2}+\cdots+B_{s} \eta_{t-s}+\zeta_{t},
$$

where the q-variate set of latent scores are a function of $k=1,2, \cdots, s$ previous latent scores, the matrices $B_{1}, \cdots, B_{s}$ represent the sequential dependencies (autoregressive coefficients and cross regression of latent variables at time $t$ on the latent variables at previous times), and $\zeta_{t}$ is the innovation vector, i.e. the new information provided by the observations at time $t$. Sequential dependencies can also be introduced in the measurements (correlations between residuals). However, the choice of model should be determined primarily by the theoretical representation of the developmental process since the various models cannot strictly speaking be compared statistically.

The above formalization, which is specific to the methodology of dynamic systems, is a central feature of current tools for modeling intraindividual variability (Chow, Ferrer, \& Hsieh, 2010; Molenaar \& Newell, 2010). The dynamic la- 
tent variable model formalizes the temporal structure of intraindividual variability by distinguishing between that which is time-dependent and that which is not (Ram \& Gerstorf, 2009). It contains a deterministic component corresponding to time-dependent dynamic processes that are subject to the influence of external variations caused by the processes themselves and, in some applications, the influence of external factors (for example, the situation or the context). These processes are associated with systematic patterns of change over time (for example, the maintenance of stability or transformations or adaptations to a change of constraint). The stochastic component of the model covers temporally unstructured dynamic characteristics, represented by the random process $\zeta_{t}$ (equation 8 ), such as the individual potential for change (for example, flexibility and innovation) or stability (for example, the robustness of the treatment). This dynamic model of intraindividual variability can be extended to several levels of analysis (Boker, Molenaar, \& Nesselroade, 2009). The hierarchical extension also suggests time-dependent changes occurring at different time scales: a real time scale associated with the dynamics of the observations and the underlying latent process(es) and a developmental time scale associated with slower dynamics. To the best of the author's knowledge, no hierarchical dynamic model has so far been applied to developmental data of this kind.

Dynamic latent variable models can also be applied to individual time series with values generated by discrete states (for example, attentional engagement of the all-or-nothing type, specific strategies, etc.). These models (from the family of hidden Markov models) are based on the definition of the state-space or space formed by all the latent states in which the individual is likely to find themselves during the process of change and on the definition of the initial state. The measurement equation of the model defines the densities of the observations describing the distributions of the observations conditional on the latent state (for example, log-normal distribution for response times, binomial distribution for accuracy). The transition equation formalizes the dynamics of the latent process with a transition matrix. The following is an example of the application of an individual time series obtained in a trial-and-error rule-learning situation (Visser et al., 2010). The task used is the Iowa gambling task, which simulates the conditions of reward and punishment in a context of uncertainty. Participants are required to choose a simultaneously losing and winning card from one of four decks placed of front of them. The win/loss ratio varies according to the cards. Two of the decks contain winning cards that will be disadvantageous in the long term (decks A and B). The two other decks contain losing cards that will be advantageous in the long term (decks C and D). The series of responses in the 200 trials of the task is adjusted using a hidden Markov model with four latent states. The beginning of the learning process in a given participant is characterized by two alternating states both associated with infrequent losses. There follow a phase of stability in a state associated with an immediate win, a short phase of instability and, finally, a stable state corresponding to optimal behavior (appro- 
priate combination of relevant dimensions).

The modeling of intraindividual variability at an individual level is a key stage before the comparisons required to identify differential invariants in developmental change. There are several methods for grouping individuals with similar dynamics by differentiating them from individuals with a differently structured intraindividual variability. For example, Nesselroade \& Molenaar (1999) used a variance homogeneity test to compare individual covariance structures. Various other methods for quantifying the similarity of individual structures (e.g. measures of distance) can also be used. The DepmixS4 R package (Visser, 2011), which can be used to estimate the parameters of the hidden Markov model, can combine several individual models informally after testing the degree of similarity between individuals in terms of successive latent states or transition probabilities. The combined use of two levels of analysis (the individual level and the differential level) increases the reliability of individual analyses while enabling the identification of groups of individuals with the same structure of intraindividual variability.

\section{Conclusion}

The differential study of intraindividual variability and change is currently reaping the benefits of recent methodological advances in longitudinal data modeling. This paper examined two major research strategies for detecting invariants in differences in developmental change.

The most common approach involves operating at a population level to study differences of behavior throughout development. Change may be seen as a common developmental trajectory characterized by quantitative interindividual variations (multilevel regression models, latent variable growth curve modeling), several qualitatively different trajectories with each trajectory being common to a subgroup (latent class growth models), or several trajectories, with each trajectory (common to individuals in the same subgroup) being characterized by quantitative interindividual variations (mixture growth models). The reduction of interindividual variability is a solution to the problem posed by a "blind" aggregation of individual data. A person-oriented approach enables the identification of differential invariants with a degree of generality, for example, a typology of intraindividual profiles in developmental change in a given area.

Though rarely used, another approach involves using the individual as the unit of analysis. Here, the assumption is that analyses of intraindividual change at a population level are ill-adapted when specific individual processes are involved. This approach requires the use of intensive measurements to individually adjust a model describing the temporal structure and dynamics of intraindividual change to suit each individual. The search for differential invariants will involve grouping individuals whose behavior reflects the same dynamic laws of intraindividual change based on certain criteria.

In short, the study of invariants in intraindividual change based on individual 
differences involves decisions related to the types of variability to be considered, the chosen methodology and the methods used, the assumption being that the approach and methods used are themselves dependent upon the preferred theoretical framework and the nature of the data. This study emphasized the choice of level of observation and the associated level of analysis. The approach used to confirm a developmental hypothesis generally involves studying differences in developmental change in a significant number of individuals. Another method of verification is the differential study of developmental change based on an individual modeling of the dynamics of intraindividual variability. However, this approach is not widely used in current research, despite its potential for improving our understanding of the development of human beings.

\section{References}

Allport, G. W. (1937). Personality: A Psychological Interpretation. New York, NY: Holt, Rinehart \& Winston.

Asendorpf, J. B. (1992). Beyond Stability: Predicting Inter-Individual Differences in Intra-Individual Change. European Journal of Personality, 6, 103-117. https://doi.org/10.1002/per.2410060204

Baltes, P. B., \& Nesselroade, J. R. (1979). History and Rationale of Longitudinal Research. In J. R. Nesselroade, \& P. B. Baltes (Eds.), Longitudinal Research in the Study of Behavior and Development (pp. 1-39). New York, NY: Academic Press.

Baltes, P. B., Reese, H. W., \& Nesselroade, J. R. (1977). Life-Span Developmental Psychology: Introduction to Research Methods. Monterrey, CA: Brooks/Cole.

Bates, J. E., \& Novosad, C. (2005). Measurement of Individual Difference Constructs in Child Development, or Taking Aim at Moving Targets. In D. M. Teti (Ed.), Handbook of Research Methods in Developmental Science (pp. 103-122). Malden, MA: Blackwell Publishing. https://doi.org/10.1002/9780470756676.ch6

Bauer, D. J., \& Curran, P. J. (2003). Distributional Assumptions of Growth Mixture Models: Implications for Overextraction of Latent Trajectory Classes. Psychological Methods, 8, 338-363. https://doi.org/10.1037/1082-989X.8.3.338

Bauer, D. J., \& Reyes, H. L. M. (2010). Modeling Variability in Individual Development: Differences of Degree or Kind? Child Development Perspectives, 4, 114-122. https://doi.org/10.1111/j.1750-8606.2010.00129.x

Bergman, L. R., Magnusson, D., \& El-Khouri, B. M. (2003). Studying Individual Development in an Inter-Individual Context. New York, NY: Erlbaum.

Boker, S. M., Molenaar, P. C. M., \& Nesselroade, J. R. (2009). Issues in Intraindividual Variability: Individual Differences in Equilibria and Dynamics over Multiple Time Scales. Psychology and Aging, 24, 858-862. https://doi.org/10.1037/a0017912

Bollen, K. A., \& Curran, P. J. (2006). Latent Curve Models: A Structural Equation Approach. Wiley Series on Probability and Mathematical Statistics, Hoboken, NJ: Wiley Intersciences.

Boscardin, C. K., Muthén, B., Francis, D. J., \& Baker, E. L. (2008). Early Identification of Reading Difficulties using Heterogeneous Developmental Trajectories. Journal of Educational Psy-Chology, 100, 192-208. https://doi.org/10.1037/0022-0663.100.1.192

Bray, B. C., Lanza, S. T., \& Collins, L. M. (2010). Modeling Relations among Discrete Developmental Processes: A General Approach to Associative Latent Transition Analysis. 
Structural Equation Modeling: A Multidisciplinary Journal, 17, 541-569.

https://doi.org/10.1080/10705511.2010.510043

Brim, O. G. Jr., \& Kagan, J. (1980). Constancy and Change: A View of the Issues. In O. G. Brim, \& J. Kagan (Eds.), Constancy and Change in Human Development (pp. 1-25). Cambridge, MA: Harvard University Press.

Bryk, A. S., \& Raudenbush, S. W. (1987). Application of Hierarchical Linear Models to Assessing Change. Psychological Bulletin, 101, 147-158. https://doi.org/10.1037/0033-2909.101.1.147

Cattell, R. B. (1952). The Three Basic Factor-Analytic Designs: Their Interrelations and Derivatives. Psychological Bulletin, 49, 499-520. https://doi.org/10.1037/h0054245

Cattell, R. B. (1964). Beyond Validity and Reliability: Some Further Concepts and Coefficients for Evaluating Tests. Journal of Experimental Education, 33, 133-143. https://doi.org/10.1080/00220973.1964.11010865

Cattell, R. B. (1966). Guest Editorial: Multivariate Behavioral Research and the Integrative Challenge. Multivariate Behavioral Research, 1, 4-23. https://doi.org/10.1207/s15327906mbr0101_1

Cattell, R. B., Cattell, A. K. S., \& Rhymer, R. M. (1947). P-Technique Demonstrated in Determining Psychophysical Source Traits in a Normal Individual. Psychometrika, 12, 267-288. https://doi.org/10.1007/BF02288941

Cattell, R. B., Eber, H. W., \& Tatsuoka, M. M. (1970). Handbook for the Sixteen Personality Factor Questionnaire. Champaign, IL: Institute for Personality and Ability Testing.

Chow, S. M., Ferrer, E., \& Hsieh, F. (2010). Statistical Methods for Modeling Human Dynamics: An Interdisciplinary Dialogue. New York, NY: Taylor and Francis.

Collins, L. M. (2006). Analysis of Longitudinal Data: The Integration of Theoretical Model, Temporal Design and Statistical Model. Annual Review of Psychology, 57, 505-528. https://doi.org/10.1146/annurev.psych.57.102904.190146

Collins, L. M., \& Lanza, S. T. (2010). Latent Class and Latent Transition Analysis for the Social, Behavioral, and Health Sciences. New York, NY: Wiley.

Curran, P. J., \& Bauer, D. J. (2011). The Disaggregation of Within-Person and Between-Person Effects in Longitudinal Models of Change. Annual Review of Psychology, 62, 583-619. https://doi.org/10.1146/annurev.psych.093008.100356

Ebner-Priemer, U. W., \& Trull, T. J. (2009). Using Experience Sampling Methods/Ecological Momentary Assessment (ESM/EMA) in Clinical Assessment and Clinical Research: Introduction to the Special Section. Psychological Assessment, 21, 457-462. https://doi.org/10.1037/a0017653

Ferrer, E., McArdle, J. J., Shaywitz, B. A., Holahan, J. N., Marchione, K., \& Shaywitz, S. E. (2007). Longitudinal Models of Developmental Dynamics between Reading and Cognition from Childhood to Adolescence. Developmental Psychology, 43, 1460-1473. https://doi.org/10.1037/0012-1649.43.6.1460

Ferrer, E., Shaywitz, B. A., Holahan, J. N., Marchione, K., \& Shaywitz, S. E. (2010). Uncoupling of Reading and IQ over Time: Empirical Evidence for a Definition of Dyslexia. Psychological Science, 21, 93-101. https://doi.org/10.1177/0956797609354084

Fiske, D. W., \& Rice, L. (1955). Intra-Individual Response Variability. Psychological Bulletin, 52, 217-250. https://doi.org/10.1037/h0045276

Gibbons, R. D., Hedeker, D., \& DuToit, S. (2010). Advances in Analysis of Longitudinal Data. Annual Review of Clinical Psychology, 6, 79-107. https://doi.org/10.1146/annurev.clinpsy.032408.153550

Kagan, J. (1971). Change and Continuity in Infancy. New York, NY: Wiley. 
Kaplan, D. (2008). An Overview of Markov Chain Methods for the Study of Stage-Sequential Developmental Processes. Developmental Psychology, 44, 457-467. https://doi.org/10.1037/0012-1649.44.2.457

Kaplan, D. (2009). Structural Equation Modeling: Foundations and Extensions (2nd Ed.). Newbury Park, CA: SAGE Publications. https://doi.org/10.4135/9781452226576

Kaplan, D., \& Walpole, S. (2005). A Stage-Sequential Model of Reading Transitions: Evidence from the Early Childhood Longitudinal Study. Journal of Educational Psychology, 97, 551-563. https://doi.org/10.1037/0022-0663.97.4.551

Kim, J., \& Cicchetti, D. (2006). Longitudinal Trajectories of Self-System and Depressive Symptoms among Maltreated and Nonmaltreated Children. Child Development, 77, 624-639. https://doi.org/10.1111/j.1467-8624.2006.00894.x

Lamiell, J. T. (1981). Toward an Idiothetic Psychology of Personality. American Psychologist, 36, 276-289. https://doi.org/10.1037/0003-066X.36.3.276

Langeheine, R. (1988). Manifest and Latent Markov Chain Models for Categorical Panel Data. Journal of Educational Statistics, 13, 299-312. https://doi.org/10.3102/10769986013004299

Langeheine, R., \& van de Pol, F. (1990). A Unifying Framework for Markov Modeling in Discrete Space and Discrete Time. Sociological Methods and Research, 18, 416-441. https://doi.org/10.1177/0049124190018004002

Lautrey, J. (1990). Sketch of a Pluralistic Model of Cognitive Development. In M. Reuchlin, J. Lautrey, C. Marendaz, \& T. Ohlmann (Eds), Cognition: The Universal and the Individual (pp. 185-216). Paris: P.U.F.

Lautrey, J. (1993). Structure and Variability: A Plea for a Pluralistic Approach to Cognitive Development. In R. Case, \&W. Edelstein (Eds.), The New Stucturalism in Cognitive Development. Theory and Research on Individual Pathways (pp. 101-114). Basel: Karger.

Lautrey, J. (2003a). A Pluralistic Approach to Cognitive Differentiation and Development. In R. J. Sternberg, J. Lautrey, \& T. Lubart (Eds.), Models of Intelligence: International Perspectives (pp.117-131). Washington DC: American Psychological Press.

Lautrey, J. (2003b). Differential Psychology Challenged by Intra-Individual Variability. In A. Vom Hofe, H. Charvin, J.-L. Bernaud, \& D. Guédon (Eds.), Differential Psychology: Research and Reflections (pp. 9-28). Rennes: P.U.R.

Lautrey, J. (2010). The Evolution of the Specificities of Differential Psychology and Its Repercussions on the Identity of the Sub-Discipline. In A. de Ribaupierre et al. (Eds.), Identities and Specificities of Differential Psychology (pp. 15-30). Rennes: P.U.R.

Lautrey, J., Mazoyer, B., \& van Geert, P. (2002). Invariants and Variabilities in Cognitive Science. Paris: Presses de la Maison des Sciences Humaines. https://doi.org/10.4000/books.editionsmsh.6672

Lazarsfeld, P. F., \& Henry, N. W. (1968). Latent Structure Analysis. Boston: Houghton Mifflin.

Martin, M., \& Hofer, S. M. (2004). Intraindividual Variability, Change, and Aging: Conceptual and Analytical Issues. Gerontology, 50, 7-11. https://doi.org/10.1159/000074382

McArdle, J. J. (1982). Structural Equation Modeling of an Individual System: Preliminary Results from “A Case Study in Episodic Alcoholism”. Unpublished Manuscript, Denver: Department of Psychology, University of Denver.

McArdle, J. J. (2001). A Latent Difference Score Approach to Longitudinal Dynamic Structural Analysis. In R. Cudeck, S. du Toit, \& D. Sörbom (Eds.), Structural Equation 
Modeling: Present and Future. A Festschrift in Honor of Karl Jöreskog (pp. 341-380). Lincolnwood, IL: Scientific Software International.

McArdle, J. J. (2009). Latent Variable Modeling of Differences in Changes with Longitudinal Data. Annual Review of Psychology, 60, 577-605. https://doi.org/10.1146/annurev.psych.60.110707.163612

McArdle, J. J., \& Epstein, D. B. (1987). Latent Growth Curves within Developmental Structural Equation Models. Child Development, 58, 110-133. https://doi.org/10.2307/1130295

Molenaar, P. C. M. (1985). A Dynamic Factor Model for the Analysis of Multivariate Time Series. Psychometrika, 50, 181-202. https://doi.org/10.1007/BF02294246

Molenaar, P. C. M. (2004). A Manifesto on Psychology as Idiographic Science: Bringing the Person Back into Scientific Psychology, This Time Forever. Measurement, 2, 201-218. https://doi.org/10.1207/s15366359mea0204_1

Molenaar, P. C. M., \& Campbell, C. G. (2009). The New Person-Specific Paradigm in Psychology. Current Directions in Psychology, 18, 112-117. https://doi.org/10.1111/j.1467-8721.2009.01619.x

Molenaar, P. C. M., de Gooijer, J. G., \& Schmitz, B. (1993). Dynamic Factor Analysis of Nonstationary Multivariate Time Series. Psychometrika, 57, 333-349. https://doi.org/10.1007/BF02295422

Molenaar, P. C., \& Newell, K. M. (2010). Individual Pathways of Change: Statistical Models for Analyzing Learning and Development. Washington DC: American Psychological Association. https://doi.org/10.1037/12140-000

Moskowitz, D. S., Russell, J. J., Sadikaj, G., \& Sutton, R. (2009). Measuring People Intensively. Canadian Psychology, 50, 131-140. https://doi.org/10.1037/a0016625

Muthén, B. (2004). Latent Variable Analysis: Growth Mixture Modeling and Related Techniques for Longitudinal Data. In D. Kaplan (Ed.), Handbook of Quantitative Methodology for the Social Sciences (pp. 345-368). Newbury Park, CA: Sage. https://doi.org/10.4135/9781412986311.n19

Muthén, B. (2008). Latent Variable Hybrids: Overview of Old and New Models. In G. R. Hancock, \& K. M. Samuelsen (Eds.), Advances in Latent Variable Mixture Models (pp. 1-24). Charlotte, NC: Information Age Publishing, Inc.

Muthén, L. K., \& Muthén, B. (1998-2017). Mplus User’s Guide. Los Angeles, CA: Muthén \& Muthén.

Nagin \& Odgers (2010). Group-Based Trajectory Modeling in Clinical Research. Annual Review of Clinical Psychology, 6, 109-138. https://doi.org/10.1146/annurev.clinpsy.121208.131413

Nagin, D. (2005). Group-Based Modeling of Development. Cambridge, MA: Harvard University Press. https://doi.org/10.4159/9780674041318

Nesselroade, J. R. (1970). Application of Multivariate Strategies to Problems of Measuring and Structuring Long Term Change. In L. R. Goulet, \& P. B. Baltes (Eds.), Life Span Developmental Psychology: Research and Theory (pp. 193-207). New York, NY: Academic Press. https://doi.org/10.1016/B978-0-12-293850-4.50013-3

Nesselroade, J. R. (1991). The Warp and the Woof of the Developmental Fabric. In R. Downs, L. Liben, \& D. S. Palermo (Eds.), Visions of Aesthetics, the Environment, \& Development: The Legacy of Joachim F. Wohlwill (pp. 213-240). Hillsdale, NJ: Lawrence Erlbaum Associates.

Nesselroade, J. R. (2004). Yes, It Is Time: Commentary on Molenaar's Manifesto. Measurement: Interdisciplinary Research and Perspectives, 2, 227-230. 
Nesselroade, J. R. (2010). On an Emerging Third Discipline of Scientific Psychology. In P. C. M. Molenaar, \& K. M. Newell (Eds.), Individual Pathways of Change: Statistical Models for Analyzing Development and Change (pp. 209-218). Washington DC: American Psychological Association. https://doi.org/10.1037/12140-012

Nesselroade, J. R., \& Ghisletta, P. (2000). Beyond Static Concepts in Modeling Behavior. In L. R. Bergman, \& R. B. Cairns (Eds.), Developmental Science and the Holistic Approach (pp. 121-135). Mahwah, NJ: Lawrence Erlbaum Associates, Publishers.

Nesselroade, J. R., \& Molenaar, P. C. M. (1999). Pooling Lagged Covariance Structures Based on Short, Multivariate Time Series for Dynamic Factor Analysis. In R. H. Hoyle (Ed.), Statistical Strategies for Small Sample Research (pp. 223-250). Thousand Oaks, CA: Sage Publications, Inc.

Nesselroade, J. R., \& Molenaar, P. C. M. (2004). Applying Dynamic Factor Analysis in Behavioral and Social Science Research. In D. Kaplan (Ed.), The Sage Handbook of Quantitative Methodology for the Social Sciences (pages). Thousand Oaks, CA: Sage Publications. https://doi.org/10.4135/9781412986311.n18

Nesselroade, J. R., \& Ram, N. (2004). Studying Intraindividual Variability: What We Have Learned That Will Help Us Understand Lives in Context. Research in Human Development, 1, 9-29. https://doi.org/10.1207/s15427617rhd0101\&2_3

Nesselroade, J. R., \& Salthouse, T. A. (2004). Methodological and Theoretical Implications of Intraindividual Variability in Perceptual Motor Performance. Journal of Gerontology: Psychological Sciences, 59B, 49-55.

https://doi.org/10.1093/geronb/59.2.P49

Nesselroade, J. R., McArdle, J. J., Aggen, S. H., \& Meyers, J. M. (2002). Dynamic Factor Analysis Models for Representing Process in Multivariate Time-Series. In D. M. Moskowitz, \& S. L. Hershberger (Eds.), Modeling Intraindividual Variability with Repeated Measures Data: Advances and Techniques (pp. 235-265). Mahwah, NJ: Lawrence Erlbaum Associates.

Quaiser-Pohl, C., Rohe, A. M., \& Amberger, T. (2010). The Solution Strategy as an Indicator of the Developmental Stage of Preschool Children's Mental-Rotation Ability. Journal of Individual Differences, 31, 95-100. https://doi.org/10.1027/1614-0001/a000017

Quinn, M. E., \& Martin, P. (1999). Intra-Individual Change and Inter-Individual Differences in Negative Mood States of Older Women. International Journal of Behavioral Development, 23, 685-701. https://doi.org/10.1080/016502599383757

Ram, N., \& Gerstorf, D. (2009). Time-Structured and Net Intraindividual Variability: Tools for Examining the Development of Dynamic Characteristics and Processes. Psychology and Aging, 24, 778-791. https://doi.org/10.1037/a0017915

Ram, N., Brose, A., \& Molenaar, P. C. M. (2013). Dynamic Factor Analysis: Modeling Person-Specific Process. In T. Little (Ed.), Oxford Handbook of Quantitative Methods (pp. 441-457). New York, NY: Oxford University Press. https://doi.org/10.1093/oxfordhb/9780199934898.013.0021

Raudenbush, S. W., \& Bryk, A. S. (2002). Hierarchical Linear Models (2nd ed.). Thousand Oaks, CA: Sage.

Reuchlin, M. (1962). Quantitative Methods in Psychology. Paris: P.U.F.

Reuchlin, M. (1978). Vicarious Processes and Individual Differences. Journal de Psychologie, 2, 133-145.

Reuchlin, M. (1999). Evolution of Differential Psychology. Paris: P.U.F.

Robinson, W. S. (1950). Ecological Correlations and the Behavior of Individuals. Ameri- 
can Sociological Review, 15, 351-357. https://doi.org/10.2307/2087176

Schmidt, F. L., Le, H., \& Ilies, R. (2003). Beyond Alpha: An Empirical Examination of the Effects of Different Sources of Measurement Error on Reliability Estimates for Measures of Individual Differences Constructs. Psychological Methods, 8, 206-224. https://doi.org/10.1037/1082-989X.8.2.206

Sliwinski, M., \& Mogle, J. (2008). Time-Based and Process-Based Approaches to Analysis of Longitudinal Data. In S. M. Hofer, \& D. F. Alwin. (Eds.), Handbook on Cognitive Aging: Interdisciplinary Perspectives (pp. 477-491). Thousand Oaks, CA: Sage Publications. https://doi.org/10.4135/9781412976589.n28

Sterba, S. K., \& Bauer, D. J. (2010). Matching Method with Theory in Person-Oriented Developmental Psychopathology Research. Development \& Psychopathology, 22, 239-254. https://doi.org/10.1017/S0954579410000015

Van de Pol, F., \& Langeheine, R. (1990). Mixed Markov Latent Class Models. In C. C. Clogg (Ed.), Sociological Methodology (pp. 213-247). Oxford: Blackwell. https://doi.org/10.2307/271087

Van Dijk, M., \& Van Geert, P. (2007). Wobbles, Humps and Sudden Jumps: A Case Study of Continuity, Discontinuity and Variability in Early Language Development. Infant and Child Development, 16, 7-33. https://doi.org/10.1002/icd.506

Vergnaud, G. (2007). Scheme Concept Definitions. Recherches en Education, 4, 17-22.

Visser, I. (2011). Seven Things to Remember about Hidden Markov Models: A Tutorial on Markovian Models for Time Series. Journal of Mathematical Psychology, 55, 403-415. https://doi.org/10.1016/j.jmp.2011.08.002

Visser, I., Jansen, B., \& Speekenbrink, M. (2010). A Framework for Discrete Change. In P. C. Mo-lenaar, \& K. M. Newell (Eds.), Individual Pathways of Change: Statistical Models for Analyzing Learning and Development (pp. 109-123). Washington DC: American Psychological Association. https://doi.org/10.1037/12140-007

von Eye, A. (2010). Developing the Person-Oriented Approach: Theory and Methods. Development \& Psychopathology, 22, 277-285. https://doi.org/10.1017/S0954579410000052

von Eye, A., \& Bergman, L. R. (2003). Research Strategies in Developmental Psychopathology: Dimensional Identity and the Person-Oriented Approach. Development \& Psychopathology, 15, 553-580. https://doi.org/10.1017/S0954579403000294 